\title{
Cost-analysis of Treatment of Patients with Acute Myeloid Leukemia
}

\author{
Vahid Alipour ${ }^{1}$, Soroush Rad ${ }^{2, * *}$, Fateme Mezginejad ${ }^{3}$, Reza Jahangiri ${ }^{4}$, Rafat Bagherzadeh ${ }^{5}$, Shahin \\ Nargesi (iD) ${ }^{1,}$ and Seyed Asadollah Mousavi (iD) ${ }^{2}$
}

\author{
${ }^{1}$ Health Management and Economics Research Center, Health Management Research Institute, Iran University of Medical Sciences, Tehran, Iran \\ ${ }^{2}$ Hematology, Oncology, and Hematopoietic Stem Cell Transportation Research Center, Tehran University of Medical Sciences, Tehran, Iran \\ ${ }^{3}$ Transfusion Research Center, High Institute for Research and Education in Transfusion Medicine, Tehran, Iran \\ ${ }^{4}$ Health Management and Economics Research Center, Iran University of Medical Sciences, Tehran, Iran \\ ${ }^{5}$ Department of Foreign Languages, School of Health Management and Information Sciences, Iran University of Medical Sciences, Tehran, Iran \\ "Corresponding author: Health Management and Economics Research Center, Health Management Research Institute, Iran University of Medical Sciences, Tehran, Iran. Email: \\ nargesi.s@iums.ac.ir \\ "Corresponding author: Hematology, Oncology, and Hematopoietic Stem Cell Transportation Research Center, Tehran University of Medical Sciences, Tehran, Iran. Email: \\ srad@sina.tums.ac.ir
}

Received 2020 September 18; Revised 2021 June 16; Accepted 2021 June 23.

\begin{abstract}
Background: Acute myeloid leukemia (AML) is the second common leukemia (5.18\%)and the third deadliest leukemia in Iran. Moreover, it is the fifth prevalent cancer in the world, which involves $8 \%$ of all cancers.

Objectives: The aim of this study was to calculate direct medical and non-medical costs of AML in 2019.

Methods: The present retrospective-descriptive analysis was conducted on 192 patients with AML aged 19 to 70 years from 2016 to 2018. The data were collected from hospital records and interviews with experts. The bottom-up micro-costing approach and payer perspective was considered for cost analysis status. The relationship of affective variables was investigated, using nonparametric tests, including Mann-Whitney and Kruskal-Wallis tests. Costs were divided into costs of diagnosis, hospitalization, medication, nursing, visit and consultation, operating room, and medical supplies. The data were described by mean \pm standard deviation and reported by percentage and also analyzed by SPSS 11 software.

Results: According to the findings, the average age of all patients was 43.91 years and $55.7 \%$ of the patients were male. The highest and the lowest diagnostic costs were associated with laboratory tests at $\$ 1656459.48$ and ultrasound charges $\$ 4229.46$, respectively. The total direct medical costs per patient were $\$ 1056624.78$ with an average of $\$ 4846.90$ and the cost of medication included $36 \%$ of the total costs. The direct medical and non-medical costs were $\$ 10485488.48$ and $\$ 487522.87$, respectively

Conclusions: Costs of AML treatment were estimated to be $\$ 1056624.78$. Finally, it can be concluded that the cost of AML in Iran is much cheaper than that compared to other countries and also due to hidden subsidies from the public sector, the payment from the patient's pocket is very small.
\end{abstract}

Keywords: Acute Myeloid Leukemia, Costs Analysis, Direct Medical Cost, Direct Non-medical Cost, Iran

\section{Background}

Leukemia accounts for $8 \%$ of all human cancers and about $7 \%$ of all deaths from malignancies; it ranks 14 th in cancer types around the world and 7th in Iran (1-4).

The prevalence of AML is approximately 3.2 per 1000 000 people, which increases with age; so, it is estimated to increase to 1.7 by age 65 and, then, to 16.2. The mortality rate from AML under the age of 10 is 0.5 and 20 per 100 000 people in the 9 th decade of life (5).

AML is more common in the United States, Australia, and Western Europe, and it is more common in females. American men have the highest numbers compared to other countries. AML is the most common type of leukemia in adults, and it is more commonly seen in older people than in youth and even adolescents. The average age of AML is about 65 years. Mortality rates in AML depend on factors such as age, sex, and race, and these factors may be affected separately. For example, death rates in the United States are more related to age. Mortality in whites is also much higher than in blacks. Of course, the reasons for these gender and racial differences have not been identified (6).

Acute myeloid leukemia (AML) is the second common leukemia (\%18.5) and the third deadliest leukemia in Iran. It is the most common acute leukemia in adults and people over 60 years of age and is more prevalent in men than women $(2,7)$. In our country, the estimated number of new cases of Leukemia were 5437 and 3711 death numbers and 
the age-standardized (World) incidence rate is 14.5 per 100 000 in 2018 (4).

AML is a clonal disorder of hematopoietic stem cells (8) and its risk factors vary from congenital and hematological disorders to environmental exposures and chemotherapeutic agents (9). Although some studies on the geographic patterns of the disease indicated more AML prevalence in North America, Western Europe, and Australia, it is one of the main health problems all over the world (10).

Even though recent advancements in therapeutic approaches increased the cure rate up to $15 \%$ in patients older than 60 years and about $40 \%$ in patients below 60 years of age, AML is a poor prognosis disease especially in the elderlies, and about 70\% of deaths occur in patients aged 65 years or older within 1 year following diagnosis $(3,10)$.

Treatment strategies in AML patients, based on their risk-stratification, involve standard or increased therapeutic regimen intensity, consolidation therapy, or hematopoietic stem cell transplantation (3).

Therefore, induction therapy (combination of cytarabine [100 - $200 \mathrm{mg} / \mathrm{m}^{2} /$ day for 7 days] and daunorubicin [ $45-90 \mathrm{mg} / \mathrm{m}^{2} /$ day for 3 days, named as the $7+3$ regimen"]) starts as the first step of the treatment procedure to achieve complete remission, and in the case of favorable response the procedure, it should be followed by consolidation therapy. However, there is a risk of resistance or relapse in patients undergoing stem cell transplantation $(2,8)$. Therefore, a precise evaluation of prognosis factors is essential for risk-stratification and therapy management and avoiding wrong treatment decisions.

Moreover, the new drugs and their increasing expenses, the advancement in technology, and limitations in health-care services, such as financial and human resources necessitate cost estimation studies to provide clear insights into the current situation and choose appropriate therapeutic agents.

Cost-of-illness studies are intended to identify the economic effects of a disease on society (economic stress). These descriptive studies seek to determine the type and amount of costs and problems that arise in a health care system and show their economic burden. Cost forecasting is essential for planning to provide services within the budget and to prioritize various interventions in health care systems (11). Given the limited financial and human resources to provide health care in different sectors (12), cost evaluation studies can be a valuable tool to consider the economic burden imposed by disease (11) and facilitate planning and prioritization for the management of this disease in the future. The present study is a cost evaluation study.

Among the costs that are considered in disease, cost studies are direct costs. Direct costs are divided into two types of direct medical costs and direct non-medical costs (13). Direct treatment costs refer to costs that are incurred directly in connection with the provision of medical services to patients. The second group is direct non-medical costs. These costs are auxiliary resources that patients pay in exchange for medical services, such as the cost of out-oftown transportation or intra-city transportation to reach specialized treatment centers, the cost of home care, all of which are paid from the patient's pocket (14).

\section{Objectives}

Although the assessment of cost analysis of AML treatment is complicated, it offers valuable information for healthcare providers, health professionals, and policymakers about the effective use of resources in AML management and improvement of community health standards. As stated earlier, AML is among the most common cancers in Iran; therefore, this study was conducted to calculate its cost analysis (direct medical and non-medical costs) in 2019.

\section{Methods}

This retrospective-descriptive study was conducted between January 2016 and December 2018. The population consisted of 290 patients aged 19 to 70 years with AML in Shariati Hospital in Tehran (the most prestigious medical, research, and educational centers in the country and one of the 4 most important stem cell transplant centers in the world, which admits patients from the entire country). Moreover, the obtained results can be generalized to the whole country, since the tariff for medical services is the same throughout Iran. The data were collected from hospital records and interviews with experts, based on which 192 patients were included in the study because they met the inclusion criteria. The inclusion criteria include patients, whose information during all stages of treatment was completely recorded. The exclusion criteria included patients under 19 years of age and those aged 70 years or older, who were unable or unwilling to answer questions. Also, some patients were excluded from the study, such as the children, due to their different treatment protocol, as well as patients, whose information was not recorded in the information system or their treatment period was not completed either due to death or referral.

The bottom-up approach was used for the cost analysis. In the bottom-up approach, the average cost per patient is first calculated and, then, multiplied by the total number of patients. To calculate the cost per patient, the various 
components of the cost are identified and the cost generated is measured for each component. For example, to calculate the total cost of patients' travel, the average number of trips per patient is multiplied by the average cost of each trip and, then, multiplied by the total number of patients (15).

All hospital-related costs linked to AML treatment were included in the cost analysis. Since those patients are insured, costs are mainly paid by health insurance.

Direct medical cost data were abstracted from inpatient and outpatient medical records; thus, it included the costs of diagnosis, hospitalization, nursing, operating room, visits, chemotherapy, medication, and medical supplies. Diagnostic costs consist of the cost of pathology, laboratory tests, consulting, radiology, and ultrasound. The questionnaire was used as a data collection tool for calculating direct non-medical costs in this study. Content validity was used to determine the scientific validity of the questionnaire so that the questionnaire was prepared and confirmed by scientific texts and opinions of some professors of medical universities. Before collecting data, the questionnaire was piloted on some patients and the ambiguities of the questionnaire were identified and resolved. The face-to-face interviews or telephone interviews with patients or their companions were performed to ask about the imposed costs, such as travel and patient care costs at home. In this study, costs were calculated and evaluated based on the payer's perspective. Accordingly, all costs incurred in managing the disease, regardless of who is paying (patient, insurance, or health system), were considered and calculated. However, in this study, only the direct treatment and non-treatment costs of AML were evaluated.

The demographic characteristics and types of costs were described by the mean, standard deviation (SD), and reported by percentage also analyzed by SPSS 11 software. Because the sample size was 192, the Shapiro-Wilk criterion was used. Total costs were taken as the dependent variable and demographic information, length of stay (LOS), costs of hospitalization, medication, diagnostic, and other costs were the independent variables. Differences between gender and costs were evaluated by the Mann-Whitney test, and to assess the costs differences between age groups and LOS, Kruskal-Wallis test was used. For the sake of international comparison (16), all costs converted into United States dollars (USD\$) based on the average exchange rate in 2019.

\section{Results}

According to the findings, $55.7 \%$ of patients were male and $44.3 \%$ were female. Also, the mean age of the patients was 43.91 ( \pm 13.02 ) with the age range of 19 to 70 years;
$68.3 \%$ of the patients had an academic degree, $96.8 \%$ were insured, and the share of insurance was $54 \%$. The average monthly income was $\$ 80.17$ ( \pm 11 ) (Table 1).

\begin{tabular}{|c|c|c|c|}
\hline Variables & Mean \pm SD & $\begin{array}{c}\text { Relative Frequency } \\
(\%)\end{array}$ & P-Value \\
\hline Gender & & 30.7 & \\
\hline Male & & 55.3 & \\
\hline Female & & 44.7 & \\
\hline Age group & $43.91 \pm 13.02$ & & 0.428 \\
\hline $19-28$ & & 16.1 & \\
\hline $29-38$ & & 19.8 & \\
\hline $39-48$ & & 21.4 & \\
\hline $49-58$ & & 26.6 & \\
\hline $59-68$ & & 16.8 & \\
\hline $69-78$ & & 1.6 & \\
\hline \multicolumn{4}{|l|}{ Education } \\
\hline Academic & & 31.7 & \\
\hline $\begin{array}{l}\text { Non- } \\
\text { academic }\end{array}$ & & 68.3 & \\
\hline $\begin{array}{l}\text { Medical } \\
\text { insurance }\end{array}$ & & 96.8 & \\
\hline $\begin{array}{l}\text { Average monthly } \\
\text { income }\end{array}$ & $80.17 \pm 11$ & & \\
\hline LOS & $76.98 \pm 36.385$ & & $<0.001$ \\
\hline $1-29$ & & 11.5 & \\
\hline $30-60$ & & 22.4 & \\
\hline $61-90$ & & 35.9 & \\
\hline $91-120$ & & 11.5 & \\
\hline$>120$ & & 18.8 & \\
\hline
\end{tabular}

Abbreviation: SD, Standard deviation.

The results of the Mann-Whitney test showed no significant differences between men and women in total costs, costs of hospitalization, medication, diagnostic and other costs $(\mathrm{P}>0.05)$.

Patients were classified into 6 age groups; $26.6 \%$ of patients were in the age group of 49 to 58 years, $21.4 \%$ were in the age group of 39 to 48 years, and the 69 to 78 age group had the lowest frequency (1.6\%) compared to other groups. Also, $19.8 \%$ of patients in the 29 to 38 age group, $16.1 \%$ in the 19 to 28 age group, and $16.8 \%$ of patients were classified in the age group of 59 to 68 years.

Based on the results of the Kruskal-Wallis test, there is not a significant difference between the age of groups and in total costs, hospitalization, medication, diagnostic, and others cost $(\mathrm{P}>0.05)$.

The mean length of hospitalization was $76.98 \pm 36.38$ 
days. The lowest and longest hospitalizations were 15 and 172 days, respectively; $11.5 \%$ of patients with hospital stay duration of 1 to 29 and 91 to 120 days, and $35.9 \%$ of patients had 61 to 90 hospitalization days. Also, $22.4 \%$ and $18.8 \%$ of patients had 30 to 60 and more than 120 hospitalization days, respectively (Table 1 ).

According to the Kruskal-Wallis test results, costs of hospitalization, medication, diagnostic costs, and other costs as well as total cost based on the length of hospitalization days have been significantly different $(\mathrm{P}<0.05)$, and this difference is greater with increasing of hospital stay duration. The difference in the cost of medication based on length of stay in hospital was more than other fees. Then, the cost of the diagnostic ranked second.

The components of the direct medical costs of AML are presented in Table 2. Diagnostic costs include the cost of radiography, consulting, radiology, sonography, laboratory testing, and pathology. The highest diagnostic costs were related to laboratory tests $\$ 1656459.48$ followed by radiology costs $\$ 110144.40$ and counseling costs $\$ 107194.37$. Moreover, laboratory and radiological services were performed for all patients. The cost of pathology services was $\$ 86082.94$. The lowest diagnostic costs were related to radiography at $\$ 61949.41$ and ultrasound at $\$ 4229.46$.

The total cost per patient was $\$ 1056624.78$ with an average of $\$ 4846.90$. The cost of medication includes $36 \%$ of the total direct medical costs followed by hospitalization (31\%) and diagnostic (20\%) costs.

The direct non-medical costs of AML in Iran in 2019 were demonstrated in Table 3. Telephone interviews revealed that most of the patients needed care during or after hospitalization. The average home care days were 64 days. Patients' care often was performed by their couples or a family member, while in a few cases, those were conducted by a hired nurse. In addition, the average number of transportations were approximately 20 trips. The average cost of transportation and home caring were $\$ 486.69$ and \$2052.50, respectively.

\section{Discussion}

The aim of this study was to determine the costanalysis of treating AML. The results of our study show that the largest share of the costs of AML treatment, like other types of leukemia, has been due to the hospital and medication costs. The highest rate of total in-hospital costs was related to medication (38\%) followed by the cost of hospitalization (31\%). According to the results, the average cost of medication and hospitalization was $\$ 18216.42$ and $\$ 14753.51$ in 2019. These findings are consistent with those of the study by Hayati et al., who found hospitalization (40.3\%) and medication costs (26.1\%) as the highest direct medical expenses (17). Similarly, in the study of Daroudi et al., the total direct medical costs of chronic myeloid leukemia in Iran was $\$ 23089323,97.3 \%$ of which was spent on medicine (18). The results of Menzin et al.'s study indicated that $84 \%$ of direct costs were related to hospitalization costs (19).

Based on the findings, the diagnostic costs accounted for $20 \%$ of total in-hospital costs, $80 \%$ of which was related to laboratory tests during treatment. These are in line with the findings of the study by Davari et al., who studied the direct therapeutic costs of acute lymphocytic leukemia in children aged 1 to 15 years in Isfahan Province and found that the highest diagnostic cost was associated with laboratory testing costs with an average of $\$ 103.22$ (20).

Moreover, the findings demonstrated that the average cost of AML for each patient was estimated to be $\$ 48098.57$ in 2019 and the share of the patient was $\$ 4846.90$, while in Hagiwara et al.'s study, the average total cost per patient was $\$ 386077$ (21). Moreover, the results of the study of Menzin et al. (19) indicated that costs for adults of AML were $\$ 62070.83$ (in 2019 US dollars). Finally, it can be concluded that most of the treatment costs for AML patients in Iran have been provided by insurance organizations and the government; so, the payment from the patient's pocket is very small. The high share of drug costs shows that chemotherapy drugs are one of the main reasons for the increase in out-of-pocket payments.

Most studies have only calculated direct medical costs and have not considered direct non-medical costs but in the study of Hayati et al., the non-medical direct cost was $\$ 1688.9$ that the largest share belonged to transportation at $\$ 675.5$ (17).

The diagnosis of AML in patients is associated with frequent hospitalizations, frequent outpatient visits, and significant use of expensive drugs that lead to higher costs for these patients $(22,23)$. Also, the disease progression and the increase in patient care resulted in cost increases. On the other hand, the need for intensive care also increases the cost of hospitalization. Up to $26 \%$ of patients need to be admitted to the intensive care unit $(23,24)$ and for those aged 60 and over, admissions to the intensive care unit will increase by $30.1 \%$. In addition, frequent hospitalization and outpatient visits in the first year after diagnosis is an important factor in the cost of AML (23-25). According to the results of our study, hospitalization and drug costs had the largest share of direct medical costs and $35.9 \%$ of patients had a length of hospital stay of 90 to 61 days and the average length of hospital stay in patients was 76.98 days.

In our study, the transportation cost was $\$ 93443.59$ and due to the greater need of patients for home care, the highest direct non-medical costs belonged to home care costs at $\$ 394079.28$. 


\begin{tabular}{|c|c|c|c|c|}
\hline Type of cost & Sum Cost & Cost(Mean) & SD & Total cost (\%) \\
\hline Hospitalization & 3219105.21 & 14753.51 & 4967 & 0.31 \\
\hline Medication & 3971178.98 & 18216.42 & 9872 & 0.38 \\
\hline Visit \& consultation & 434945.82 & 1995.16 & 256 & 0.04 \\
\hline Medical supplies & 453217.65 & 2078.98 & 318 & 0.04 \\
\hline Nursing & 209071.04 & 959.04 & 211 & 0.02 \\
\hline Diagnostic services & 2065075.49 & 9367.15 & 916 & 0.20 \\
\hline Total & 10485488.48 & 48098.57 & 13067 & 100 \\
\hline Share of patients & 1056624.78 & 4846.90 & 675 & \\
\hline
\end{tabular}

Abbreviation: SD, Standard deviation.

\begin{tabular}{lccc}
\hline \multicolumn{4}{l}{ Table 3. The Direct Non-Medical Costs Due to Acute Myeloid Leukemia in $2019(\$)$} \\
\hline Cost Type & Travelling Costs & Home Care Costs & Total \\
\hline $\begin{array}{l}\text { Direct } \\
\text { non-medical costs }\end{array}$ & & & \\
$\quad$ Per patient & 486.69 & 2052.50 & 2539.18 \\
\hline Total & 93443.59 & 394079.28 & 487522.87 \\
\hline
\end{tabular}

Information on health care costs can help health system managers and officials to distribute financial resources efficiently and effectively and make appropriate decisions about prevention or treatment programs and budgeting. In addition, the cost of this disease can be used as an introduction to other economic studies in this field.

The present research was the first study to examine the costs of AML patients in Iran; however, it should be noted that the calculated costs may be less than the actual amount of costs of the disease, which could be attributed to the defects in patient information registration and incomplete registration of processes and care costs by employees.

Another limitation of this study was the lack of cooperation between some patients and their companions. In this case, an attempt was made to obtain their consent for the interview by talking to patients and their companions, explaining the importance of this issue and the results of the study, which help improve patients' well-being. Another limitation of the study was the error of reminding patients and their families in expressing the type and amount of costs, which was tried to reduce this error as much as possible by preparing a comprehensive questionnaire to remind costs from the time of the interview.

\subsection{Conclusions}

The costs of AML treatment in patients aged 19 to 70 years amounted to $\$ 10485488.48$, which due to hidden subsidies in governmental sectors, are much less than the actual costs spent for these patients. Inpatient and medication costs of AML account for the highest percentage of expenses and are important factors in the cost of treatment of these patients. Finally, it can be concluded that the direct medical cost of AML in Iran is much cheaper than that compared to other countries and also the payment from the patient's pocket is very small.

\section{Acknowledgments}

This study was an extract from a research project with the Code of Ethics IR.IUMS.REC .1397.1360 from Iran University of Medical Sciences. The project was conducted and supported by the Health Management and Economics Research Center, Iran University of Medical Sciences, Tehran, Iran. We would like to thank all the personnel and medical staff at Shariati Hospital Medical Education Center for their cooperation and assistance in conducting the research.

\section{Footnotes}

Authors' Contribution: SR and SHN initiated and designed the study. SHN and FM drafted the manuscript. SR , AM, RJ,SHN, performed the data analysis. All of the authors contributed to the revision of the manuscript and approved the final version.

Conflict of Interests: The authors declare that they have no conflict of interest.

Ethical Approval: This article does not contain any studies with human participants or animals performed by any of the authors. 
Funding/Support: This study was part of a research project funded by the health management and economics research center affiliated to Iran University of Medical Sciences (Grant no: 97-4-48-14003 and ethical code: IR.IUMS.REC .1397.1360).

\section{References}

1. Rezapour A, Nargesi S, Mezginejad F, Rashki Kemmak A, Bagherzadeh $\mathrm{R}$. The economic burden of cancer in Iran during 1995-2019: A systematic review. Iranian Journal of Public Health. 2021;50(1):35-45. doi: 10.18502/ijph.v50i1.5070.

2. Estey EH. Acute myeloid leukemia: 2014 update on risk-stratification and management. Am J Hematol. 2014;89(11):1063-81. doi: 10.1002/ajh.23834. [PubMed: 25318680].

3. De Kouchkovsky I, Abdul-Hay M. 'Acute myeloid leukemia: A comprehensive review and 2016 update'. Blood Cancer J. 2016;6(7). e441. doi: 10.1038/bcj.2016.50. [PubMed: 27367478]. [PubMed Central: PMC5030376].

4. WHO. Global Cancer Observatory. 2020. Available from: https: //gco.iarc.fr/today/data/factsheets/populations/900-world-factsheets.pdf.

5. Greer PJ, Foerster J, Lukens JN. Wintrobes clinical hematology. Acute and chronic myeloid leukemia.10th ed. Lippincott Williams \& Wilkins; 2005.

6. Redaelli A, Lee JM, Stephens JM, Pashos CL. Epidemiology and clinical burden of acute myeloid leukemia. Expert Rev Anticancer Ther. 2003;3(5):695-710. doi: 10.1586/14737140.3.5.695. [PubMed: 14599092].

7. Seyedesmaeili N, Onsory KH. Expression of JAK2 and microRNA$216 \mathrm{a}$ in patients with acute myeloid leukemia. Iran South Med J. 2018;21(3):186-96.

8. Estey EH. Acute myeloid leukemia: 2019 update on risk-stratification and management. Am J Hematol. 2018;93(10):1267-91. doi: 10.1002/ajh.25214. [PubMed:30328165].

9. Vakiti A, Mewawalla P. Cancer, acute myeloid leukemia (AML, erythroid leukemia, myelodysplasia-related leukemia, BCR-ABL chronic leukemia). Treasure Island (FL): StatPearls Publishing; 2020.

10. Medeiros BC, Chan SM, Daver NG, Jonas BA, Pollyea DA. Optimizing survival outcomes with post-remission therapy in acute myeloid leukemia. Am J Hematol. 2019;94(7):803-11. doi: 10.1002/ajh.25484. [PubMed: 30945331]. [PubMed Central: PMC6593671].

11. Jefferson T, Demicheli V, Mugford M, Baranpourian F. Economic evaluation of health care services. Tehran, Iran: Higher Institute for Social Research; 2007.

12. Center to Reduce Cancer Health Disparities. Economic costs of cancer health disparities: Summary of meeting proceedings. Bethesda, Md: Department of Health \& Human Services, National Institutes of Health, National Cancer Institute; 2007.

13. Rice DP. Cost of illness studies: what is good about them? Inj Prev. 2000;6(3):177-9. doi: 10.1136/ip.6.3.177. [PubMed: 11003181]. [PubMed Central: PMC1730654].
14. Mohammadzadeh M, Derafshi H, Ghari T. The estimation of economic burden of hepatitis C virus infection in Iran. Iran J Public Health. 2018;47(10):1575-82. [PubMed: 30524989]. [PubMed Central: PMC6277710].

15. Segel JE. Cost of illness studies-A primer: RTI international, RTI-UNC center of excellence in health promotion economics. North Carolina US. 2006.

16. Nargesi S, Abutorabi A, Alipour V, Tajdini M, Salimi J. Costeffectiveness of endovascular versus open repair of abdominal aortic aneurysm: A systematic review. Cardiovasc Drugs Ther. 2021;35(4):82939. doi: 10.1007/s10557-020-07130-6. [PubMed: 33559809].

17. Hayati H, Kebriaeezadeh A, Ehsani MA, Nikfar S, Mehrvar A. Costanalysis of treatment of pediatrics acute lymphoblastic leukemia based on ALL-BFM protocol. Int I Pediatr. 2016;4(9):3381-9. doi: 10.22038/ijp.2016.7370.

18. Daroudi R, Mirzania M, Nikravanfard N, Sadighi S, Sedighi Z, Zendehdel K. Estimation of the prevalence and direct medical costs of chronic myeloid leukemia in the I.R. of Iran in the era of tyrosine kinase inhibitors. Asia Pac J Clin Oncol. 2017;13(5):e416-22. doi: 10.1111/ajco.12470. [PubMed: 26990676]

19. Menzin J, Lang K, Earle CC, Kerney D, Mallick R. The outcomes and costs of acute myeloid leukemia among the elderly. Arch Intern Med. 2002;162(14):1597-603. doi: 10.1001/archinte.162.14.1597. [PubMed: 12123403].

20. Davari M, Moafi A, Yarmohammadian MH, Haghighi EK. The direct medical costs of acute lymphocytic leukemia (ALL) in children in Isfahan province. Health Inf Manage. 2015;11(7):1047-57.

21. Hagiwara M, Sharma A, Chung KC, Delea TE. Healthcare resource utilization and costs in patients with newly diagnosed acute myeloid leukemia. J Med Econ. 2018;21(11):1119-30. doi 10.1080/13696998.2018.1513847. [PubMed: 30122084].

22. Preussler JM, Meyer CL, Mau LW, Majhail NS, Denzen EM, Edsall $\mathrm{KC}$, et al. Healthcare costs and utilization for patients age 50 to 64 years with acute myeloid leukemia treated with chemotherapy or with chemotherapy and allogeneic hematopoietic cell transplantation. Biol Blood Marrow Transplant. 2017;23(6):1021-8. doi: 10.1016/j.bbmt.2017.02.017. [PubMed: 28263920]. [PubMed Central: PMC5497312]

23. Bell JA, Galaznik A, Farrelly E, Blazer M, Murty S, Ogbonnaya A, et al. Economic burden of elderly patients with acute myeloid leukemia treated in routine clinical care in the United States. LeukRes. 2018;71:27-33. doi:10.1016/j.leukres.2018.06.010. [PubMed: 29944984].

24. Halpern AB, Culakova E, Walter RB, Lyman GH. Association of risk factors, mortality, and care costs of adults with acute myeloid leukemia with admission to the intensive care unit.JAMA Oncol.2017;3(3):374-81. doi:10.1001/jamaoncol.2016.4858. [PubMed: 27832254]. [PubMed Central: PMC5344736].

25. Wang $\mathrm{R}$, Zeidan $\mathrm{AM}$, Halene $\mathrm{S}, \mathrm{Xu} \mathrm{X}$, Davidoff AJ, Huntington $\mathrm{SF}$, et al. Health care use by older adults with acute myeloid leukemia at the end of life. J Clin Oncol. 2017;35(30):3417-24. doi: 10.1200/JCO.2017.72.7149. [PubMed: 28783450]. [PubMed Central: PMC5648174]. 